Relations industrielles

Industrial Relations

\title{
Décisions rendues par le Conseil canadien des relations du travail
}

\section{Daniel Lavery}

Volume 37, numéro 2, 1982

URI : https://id.erudit.org/iderudit/029265ar

DOI : https://doi.org/10.7202/029265ar

Aller au sommaire du numéro

\section{Éditeur(s)}

Département des relations industrielles de l'Université Laval

\section{ISSN}

0034-379X (imprimé)

1703-8138 (numérique)

Découvrir la revue

\section{Citer cet article}

Lavery, D. (1982). Décisions rendues par le Conseil canadien des relations du travail. Relations industrielles / Industrial Relations, 37(2), 438-450.

https://doi.org/10.7202/029265ar

Tous droits réservés (C) Département des relations industrielles de l'Université Laval, 1982
Ce document est protégé par la loi sur le droit d'auteur. L'utilisation des services d'Érudit (y compris la reproduction) est assujettie à sa politique d'utilisation que vous pouvez consulter en ligne.

https://apropos.erudit.org/fr/usagers/politique-dutilisation/ 


\section{Décisions rendues par le Conseil canadien des relations du travail}

\section{Les employés en période de probation et le droit à l'arbitrage}

Le Conseil a accueilli une plainte formulée en vertu de l'article 136.1 du Code à l'encontre d'un syndicat ayant failli à son devoir de représentation juste et équitable à l'endroit d'un employé ayant fait l'objet d'un congédiement alors qu'il était en période de probation. En outre, il a déclaré invalide, parce que contraire à l'article 155 du Code, une clause de la convention collective sur laquelle le syndicat se basait prohibant le recours à la procédure de règlement des griefs et d'arbitrage à l'égard des employés en période de probation au cas de congédiement et de mise à pied.

Larry Elliston et les Métallurgistes unis d'Amérique et Boréal Navigation Limitée, Québec (Québec).

Dossier 745-917; décision du 18 janvier 1982 (no 364); Panel du Conseil: Me Claude Foisy, Vice-président, Messieurs Jacques Archambault et Victor E. Gannon, Membres.

\section{FAITS}

Le plaignant, Larry Elliston, était engagé le 24 septembre 1980 à titre de « $3^{\mathrm{e}}$ officier» à bord du navire Sunemerillon, propriété de la compagnie Boréal Navigation laquelle était liée par une convention collective intervenue le 15 août 1979 avec les Métallurgistes unis d'Amérique.

Monsieur Elliston, membre du syndicat et inclus dans l'unité de négociation, ne fut à l'emploi de la compagnie que jusqu'au 10 octobre 1980 , date à laquelle il fut congédié par le capitaine du vaisseau qui le pria de quitter le navire alors que ce dernier faisait escale à Liverpool en Nouvelle-Écosse. Il formula aussitôt un grief auquel il ne fut pas donné suite, ni par l'employeur, ni par le syndicat. Ce dernier l'avisa en effet que, n'ayant été à l'emploi de la compagnie que pour une période moindre de vingt-cinq (25) jours, il était classé employé en période de probation. À ce titre, de par la convention collective, il n'avait pas droit de recourir à la procédure de règlement des griefs et d'arbitrage, en cas de congédiement ${ }^{1}$. Monsieur Elliston formula alors, le 12 janvier 1981, une plainte en vertu de l'article 136.1 du Code à l'encontre de son syndicat réclamant que son grief soit soumis à l'arbitrage.

* Cette chronique a été rédigée par Daniel LAVERY, avocat, adjoint légal au président du Conseil canadien des relations du travail.

1 Cette clause se lisait comme suit:

«9.02 L'ancienneté s'acquiert après une période de probation d'au moins vingt-cinq (25) jours avec la compagnie ou à la fin du premier voyage au long cours. Pendant cette période, l'employé est considéré à l'essai et ne peut avoir recours à la procédure de règlement des griefs en cas de congédiement ou de mise à pied. Après cette période de probation, l'ancienneté d'un employé est rétroactive à la date de son dernier embauchage à la compagnie.»

Relat. ind., vol. 37 no 2 (1982) (c) PUL ISSN 0034-379 X 


\section{QUESTIONS SOULEVÉES}

Le Conseil devait décider si une convention collective peut validement prévoir que certaines catégories de conflits de droit (par opposition à conflits d'intérêt) à l'endroit de certains employés ne pourront faire l'objet d'un grief et d'un recours à l'arbitrage au regard des prescriptions impératives de l'article 155 du Code.

La réponse à cette question soulevait par ailleurs d'autres problèmes. $\mathrm{Si}$, par exemple, une telle clause est invalide, les parties devant obligatoirement prévoir le recours à la procédure de règlement des griefs et d'arbitrage, sont-elles libres, d'autre part, de restreindre à la convention collective la définition de ce que constitue un différend de façon à exclure le cas du congédiement d'un employé en période de probation par exemple. À défaut, peuvent-elles prévoir une norme de révision des décisions de l'employeur relevant de la discrétion complète de ce dernier concernant le congédiement de tels employés sans pour autant affecter illégalement la juridiction de l'arbitre des griefs prévus à l'article 157d) du Code. Enfin, si la clause incriminée s'avère invalide, le syndicat a-t-il, de fait, contrevenu au devoir que lui impose l'article 136.1 du Code et, le cas échéant, quels sont les remèdes appropriés dans les circonstances.

\section{PRINCIPAUX MOTIFS DE DÉCISION}

\section{Validité de la clause au regard de l'article 155 du Code}

Suite à l'étude des dispositions de la convention collective, le Conseil constate qu'elle confère, d'une part, à tous les employés, qu'ils soient permanents ou en période de probation, le droit à la juste cause au cas de congédiement et établit, d'autre part, une procédure de règlement des griefs et d'arbitrage à laquelle peuvent recourir tous les employés à l'exclusion, cependant, des employés en période de probation au cas de leur congédiement. Ces derniers jouissent, par conséquent, du droit à la juste cause au cas de congédiement mais sont privés de quelques procédures en vue de faire sanctionner ce droit.

Le Conseil avait déjà indiqué, à cet égard, dans une affaire de Transair Limited ${ }^{2}$ qu'il se ralliait aux conclusions auxquelles en était arrivé le Conseil de la ColombieBritannique sur ce sujet ${ }^{3}$ et que le renvoi des employés sous probation devait être arbitrable. Constatant, cependant, que la jurisprudence subséquente n'était pas unanime sur la question ${ }^{4}$ il procède à l'étude de cette dernière et aux décisions récentes de la Cour de Division de l'Ontario ${ }^{5}$. Il élabore par la suite sa politique concernant l'interprétation des articles 154 et 155 du Code:

2 The Canadian Airline Flight Attendants Association, Local union numéro 13 and Transair (1978) 27 di 739; (1978) 2 Can LRBR 354.

3 United Steel Workers of America, Local 6536 and Cassiar Asbestos Corporation (1974) 1 Can LRBR 428 conf. par (1975) 1 Can LRBR 212.

4 Voir à ce sujet, Michael Bendel, "Labour Law - Grievance Arbitration - Can Collective Agreements Block Recourse to Arbitration?” (1981) 59 R. du B. Can. 396.

5 International Waxes Limited c. Oil, Chemical and Atomic Workers International Union (1978) 79 C.L.L.C., 14, 203; Re Toronto Hydro Electric Systems and Canadian Union of Public Employees, Local 1 (1980) 29 O.R. (2d) 18. 
L'article 154 énonce qu'une convention collective conclue par un employeur et un agent négociateur pour une unité de négociation lie non seulement ces derniers mais également "tous les employés de l'unité de négociation» (154b). Or, il découle du paragraphe $155^{l}$ que lorsque les parties conviennent de certains droits dans une convention collective, elles doivent obligatoirement prévoir une procédure de règlement des griefs et de recours à l'arbitrage pour la solution de tous les conflits ("all differences") pouvant surgir relativement à ces droits à l'égard de toutes les personnes ci-haut mentionnées liées par la convention collective («tous les conflits surgissant... entre les parties à la convention ou les employés liés par elle»).

Les parties, y compris les salariés, étant liées par ces droits, elles ne peuvent omettre de prévoir, conformément à l'obligation légale prévue par le paragraphe $155^{l} d u$ Code, une procédure de règlement des griefs pouvant surgir relativement à l'interprétation, au champ d'application, à l'application ou la présumée violation de ces derniers, non plus que restreindre à l'égard' de l'un ou l'autre de ces droits le recours prévu par la Loi à l'endroit de l'un ou l'autre des groupes d'employés compris dans l'unité.

L'intention du législateur, à cet égard, est manifeste, puisque, au paragraphe $155^{2}$, il a mentionné expressément que «le conflit doit, nonobstant toutes dispositions de la convention collective, être soumis par les parties, pour règlement définitif». ${ }^{6}$

Appliquant ces principes à la clause incriminée de la convention collective, le Conseil conclut qu'elle est invalide pour une double raison. Elle nie, en effet, d'une part, à l'égard des employés en période de probation le recours impératif prévu au paragraphe $155^{1}$ relativement au moyen de faire sanctionner le droit au critère de la cause juste et suffisante de congédiement appartenant, aux termes de la convention collective, à tous les employés de l'unité dont font également partie les employés en période de probation. D'autre part, le statut même d'employé en période de probation pouvait donner lieu à un différend au sens du Code de sorte que les dispositions impératives de la Loi relatives au mode de règlement définitif de tels griefs devaient recevoir application. En effet, dès la création, à la convention collective, d'un statut particulier pour les employés nouvellement embauchés, la possibilité d'un différend relativement à ce statut existait, de sorte que le recours à la procédure de règlement des griefs et à l'arbitrage devait être accessible.

\section{Restrictions à la définition de différend}

Puisque la convention collective doit obligatoirement prévoir une clause de règlement définitif de tous les conflits surgissant à propos de l'interprétation, du champs d'application, de l'application ou de la présumée violation de la convention collective, se pose alors la question de déterminer si les parties peuvent conventionnellement restreindre cette définition à certaines situations de faits ou, en d'autres termes, convenir que certaines situations, tel que le congédiement de certains employés, ne constitueront pas un différend. Le Conseil répond de façon péremptoire:

6 Pages 31 et 32 de la décision du Conseil. 
(...) les parties ne peuvent non plus convenir à la convention collective d'une définition différente de ce que constitue un conflit de droit au sens $d u$ paragraphe $155^{l} \mathrm{du}$ Code puisque c'est la définition statutaire prévue par le législateur qui doit prévaloir. (...)

D'autre part, le paragraphe $155^{\prime}$ du Code mentionne expressément, (...) que "tous les conflits» ("all differences") doivent être solutionnés par le recours à la procédure de règlement des griefs et d'arbitrage. (...) Il en résulte que le législateur a délibérément fait échec au régime de la.liberté contractuelle quant à la définition de ce que constitue un différend. ${ }^{7}$

\section{La norme de révision et la juridiction de l'arbitre}

Si les parties ne peuvent nier le recours à l'arbitrage à l'égard de tous droits contenus dans une convention collective conférés à quelque employé ou groupe de ces derniers compris dans l'unité de négociation et liés, aux termes de l'article $154 \mathrm{du}$ Code, par la convention collective, elles sont libres cependant de prévoir une norme de révision particulière devant lier l'arbitrage au cas de congédiement d'une certaine catégorie d'employés ${ }^{8}$. Peuvent-elles, par ailleurs, en prévoir une si faible... «qu'il n'existerait pas de différence appréciable entre l'affirmation que l'affaire est arbitrable, mais que la faculté de réviser des arbitres est inexistante, et cette autre affirmation que l'affaire n'est pas arbitrable»' ${ }^{9}$. Le Conseil répond de la façon suivante:

À cet égard, il convient de souligner que, même en présence d'une norme dans une convention collective conférant à l'employeur toute discrétion concernant le congédiement d'une certaine catégorie d'employés, il reste néanmoins que certains motifs peuvent donner ouverture à l'intervention de l'arbitre. Il en est ainsi, par exemple, en matière de promotion lorsque la convention collective prévoit une "clause d'ancienneté modifiée» du type «compétence relative» ou encore une "clause hybride» conférant à l'employeur une discrétion totale quant à l'évaluation de la compétence des postulants. (...) L'employeur ne peut, en effet, dans l'exercice de sa discrétion, faire preuve de discrimination, parti pris ou injustice grave.

(...) Le rôle qu'exercera l'arbitre en présence d'une clause dans la convention collective conférant une discrétion à l'employeur se compare alors à celui de la Cour supérieure siégeant en matière d'évocation ou d'action directe en nullité.

(...) Les mêmes principes qui se sont développés en matière de promotion sont également applicables en matière de congédiement discrétionnaire. Le rôle que jouera l'arbitre sera alors le même que celui qu'il jouerait en matière de promotion en présence d'une clause conférant à l'employeur une certaine discrétion dans l'évaluation des candidats.

(...) Nous concluons, par conséquent, que, même en présence d'une clause à la convention collective conférant à l'employeur une discrétion totale concernant le congédiement des employés en période de probation, le rôle de l'arbitre, quoique diminué, serait néanmoins réel et constituerait le gage d'un exercice sans mauvaise foi, discrimination ou arbitraire de la discrétion de l'employeur.

7 Id., pages 33 et 34 de la décision du Conseil.

8 Voir Transair, supra, no. 2, page 746., et la présente décision du Conseil, page 36.

9 Transair, Id. 
Au surplus, avant même de se rendre à l'étape ultime de l'arbitrage, il y a possibilité que l'employé, en ayant recours à la procédure interne de règlement des griefs, fasse réformer une décision prise arbitrairement à son endroit. ${ }^{10}$

L'inclusion dans une convention collective d'une clause conférant entière discrétion à l'employeur lors du congédiement d'employés en période de probation aurait-elle pour effet de porter atteinte à la juridiction de l'arbitre prévue à l'article $157 b)$ du Code. Le Conseil répond par la négative. Cet article confère, en effet, à l'arbitre une double juridiction portant d'une part, sur l'existence de la faute lui permettant d'entendre et d'évaluer la preuve des faits fautifs et, d'autre part, sur le bien fondé de la sanction lui permettant de substituer à la mesure imposée par l'employeur celle qui lui semble appropriée dans les circonstances.

L'exercice de ce pouvoir de substitution de la sanction sous-entend implicitement que l'arbitre devra évaluer la sévérité de la sanction imposée en relation avec la gravité des faits reprochés eu égard à un critère ou à une norme de révision convenu entre les parties. Or, l'article 157 n'impose nullement à ces dernières de convenir obligatoirement du critère de la juste cause. Il est donc loisible aux parties de restreindre ou de diminuer la latitude, dont jouit habituellement l'arbitre, en convenant d'un critère ou d'une norme de révision moins large que celui de la juste cause. Ce faisant, elles ne portent pas atteinte à la double juridiction de ce dernier mais restreignent les motifs donnant ouverture à son intervention. ${ }^{11}$

Notons par ailleurs, comme le mentionne le Conseil, en se référant à sa décision antérieure dans l'affaire Len Larmour et al. ${ }^{12}$, que le syndicat, en convenant d'une telle norme restrictive de révision, ne contreviendrait pas à son devoir de représentation juste et équitable découlant de l'article 136.1 du Code, lequel s'applique également dans le cadre de la négociation d'une convention collective.

En effet, en raison des principes énoncés dans l'arrêt Len Larmour (supra), le critère de la juste cause de congédiement à l'égard de certains groupes d'employés peut être abandonné au profit d'une norme plus restrictive de revision dans le contexte du processus de la négociation d'une convention collective, un syndicat jouissant à cette occasion de la latitude nécessaire lui permettant de transiger sur les intérêts des divers groupes de l'unité aux fins de maximiser les avantages qu'il peut obtenir au profit de l'ensemble des membres de l'unité. Prenant en considération la décision du Conseil dans l'affaire Oshawa Flying Club (1981) 2 Can. LRBR 95, il en résulte qu'avant le dépôt d'une requête en accréditation, les employés se conformant aux conditions prévues par l'article $61.5^{1}$ de la Division V.7 de la Partie III du Code canadien du travail relative aux normes minimales du travail jouissent du droit à la cause juste au cas du congédiement lequel est maintenu, suite au dépôt de la requête en accréditation, par le biais de l'article $124^{4}$ de la Partie V du Code et ultérieurement par celui de l'article 148(b) jusqu'à ce que, à l'occasion de la négociation d'une première convention collective, le syndicat consente à la modification de ce droit. Le Conseil mentionnait récemment à cet égard dans une affaire de la

10 Pages 37 à 41 de la présente décision du Conseil.

11 Pages 42 et 43 de la décision du Conseil.

12 Len Larmour et al. (1980) 3 Can. LRBR 407. Pour un commentaire de cette décision, voir, Daniel Lavery, Décisions rendues par le Conseil canadien des relations du travail (1982) Relations Industrielles, Volume 37, Numéro 1, Page 226. 
Banque de Nouvelle-Écosse et Union internationale des employés de commerce (Dossier 530-646; décision du 29 janvier 1982 (numéro 367)) à la page 13 de son jugement ce qui suit:

Sont également gelées aux termes de l'article 148b), toutes les dispositions statutaires qui s'appliquent aux employés visés par l'envoi de l'avis de négocier, telles que les normes statutaires de travail, les dispositions de la Charte des droits, de même que tous les droits et privilèges dont jouissent les employés au moment de l'envoi dudit avis de négocier.

\section{Infraction à l'article 136.1 du Code}

La raison à l'origine de l'inertie du syndicat intimé face au grief du plaignant reposait sur la négation du droit à l'arbitrage à l'égard des employés en période de probation contenue à la clause invalide de la convention collective. La plainte de ce dernier n'a pas fait l'objet des démarches que, n'eût été de la présence de la clause invalide, le syndicat aurait dû normalement entreprendre. Le Conseil conclut par conséquent que l'intimé a péché par un manque total de représentation contrevenant directement aux obligations découlant de l'article 136.1 du Code.

\section{Les remèdes}

Quoique le syndicat intimé ait pêché par une absence totale de représentation, il n'a évidemment pas fait preuve de mauvaise foi, de discrimination ou d'arbitraire et ne s'est pas non plus conduit de façon gravement négligente puisqu'en se basant, à tort, sur une disposition invalide de la convention collective, il a cru sincèrement ne pas avoir à représenter le plaignant. Il n'a par conséquent simplement pas eu l'occasion d'exercer son devoir de représentation à l'endroit de ce dernier. Dans ces circonstances, le Conseil n'ordonne pas le recours immédiat à l'arbitrage mais renvoie, plutôt, le dossier aux agents du syndicat affectés à l'administration des griefs afin qu'ils puissent étudier au mérite le grief du plaignant quant à son bien fondé et l'opportunité de le pousser jusqu'à l'étape ultime de l'arbitrage. L'exécutif du syndicat devra réviser la décision de ces agents en présence du plaignant lequel aura droit d'être représenté par l'avocat de son choix aux fins de soumettre des représentations.

Le Conseil prend soin d'élargir tous les délais prescrits dans la convention collective advenant le cas où l'exécutif du syndicat déciderait de porter le grief à l'arbitrage. Il donne ainsi à ce dernier l'occasion d'exercer son devoir de représentation juste et équitable. Le Conseil renvoie enfin les parties à ses décisions antérieures rendues sous l'empire de l'article 136.1 du Code concernant les critères devant les guider lors de la prise de leur décision.

\section{Le gel statutaire de l'article 148b) du Code}

Le Conseil a révisé en séance plénière sa position concernant le gel statutaire décrété par l'article 148b) du Code entrant en vigueur suite à l'envoi d'un avis de négocier par l'une des parties en vertu de l'article 146 du Code. Il a ainsi confirmé la décision antérieure rendue entre les mêmes parties par un panel présidé par Me 
Claude Foisy, Vice-Président, lequel avait retenu, semblablement à la situation prévalant, en vertu de l'article $124^{4} d u$ Code, suite au dépôt d'une demande en accréditation, la théorie du "Statut quo" ou du "Business as usual" de préférence à la théorie $d u$ "Equal partnership" dans le cadre de l'application de l'article 148b) $d u$ Code.

La Banque de Nouvelle-Écosse, Toronto (Ontario) (Succursale située au 100, rue Wellington, Sherbrooke et Plaza Rock Forest, Rock Forest (Québec), requérante, et Union internationale des employés de commerce, intimée.

Dossier 530-646; décision du 29 janvier 1982 (no 367); Membres du Conseil adhérant à la décision majoritaire: Me Marc Lapointe, c.r., Président, Me Claude H. Foisy, Vice-Président, et Monsieur James D. Abson, Me Paul-Émile Chiasson, M. Victor E. Gannon, M. Hugh R. Jamieson, Me Nicole Kean et M. Lorne E. Shaffer. Membres minoritaires du panel: Me James E. Dorsey, Vice-Président, et M. Jacques Archambault.

La Banque de la Nouvelle-Écosse a demandé la révision de cette décision en Cour fédérale, division d'appel, en vertu de l'article 28 de la Loi sur la Cour fédérale le 8 février 1982, dossier de la Cour A-62-82.

\section{FAITS}

Dans cette cause, l'Union internationale des employés de commerce avait formulé une plainte de pratique déloyale alléguant que la Banque de la Nouvelle-Écosse avait violé les dispositions de l'article $148 \mathrm{~b}$ ) du Code canadien du travail en refusant d'appliquer aux employés syndiqués des deux succursales pour lesquelles elle détenait des certificats d'accréditation, une augmentation générale de huit pour cent décrétée le 8 janvier 1980, suite à la réception, par la banque, d'un avis de négocier en vertu de l'article 146 du Code. Cette dernière avait en effet communiqué la nouvelle de cette augmentation par le directeur de chaque succursale à tous les employés de la banque, sauf à ceux qui étaient syndiqués. En se basant sur les décisions antérieures du Conseil ${ }^{13}$, elle alléguait que les conditions de travail de ces derniers étaient gelées depuis le moment de l'accréditation et que toute modification à ces dernières qui n'étaient pas le résultat de la négociation collective constituait une pratique déloyale. L'augmentation générale ne pouvait par conséquent être accordée aux employés syndiqués puisqu'elle avait été décrétée postérieurement à l'accréditation et n'avait pas fait l'objet d'une négociation. L'Union prétendait, au contraire, que cette augmentation de huit pour cent était un droit acquis constituant le point de départ des demandes monétaires qu'elle soumettait dans son projet de convention collective.

13 Service, Office and Retail Workers Union of Canada, and The Royal Bank of Canada, Kamloops and Gibsons, B.C. 27 di 701, (1978) 2 Can LRBR 159, 78 C.L.L.C. 16, 132; Service, Office and Retail Workers Union of Canada, and Bank of Nova Scotia, 28 di 901, (1978) 2 Can LRBR 181. Voir également les décisions suivantes du Conseil concernant la théorie du "Equal partnership" dorénavant caduque: Canadian Airline Pilots" Association and Air Canada 24 di 203; Union of Bank Employees, Local 2,100 et 2,104, and The Canadian Imperial Bank of Commerce 35 di 105, (1980) 1 Can LRBR 307, 80 C.L.L.C. 16,002; Bank of British Columbia and Union of Bank Employees Local 2,100, 40 di 57, (1980) 2 Can LRBR 441, 80 C.L.L.C. 16,032; Union of Bank Employees (Local 2,100) and Bank of British Columbia (1980) 3 Can LRBR 576, 81 C.L.L.C. 16,068; et Union internationale des employés de commerce et la Banque de Nouvelle-Écosse (1981) 2 Can LRBR 365, 81 C.L.L.C. 16,110. 


\section{QUESTION SOULEVÉ}

Le gel statutaire de l'article 148b) du Code entrant en vigueur suite à l'envoi d'un avis de négocier par l'une des parties conformément à l'article 146 du Code doit-il être interprété semblablement à la situation qui prévaut suite au dépôt d'une requête en accréditation en vertu de l'article $124^{4}$ du Code selon la théorie du «Statu quo" ou du "Business as usual" de préférence à la théorie dite du "Equal partnership"'?

\section{DÉCISION DU PREMIER APPEL}

Lors de la décision originale ${ }^{14}$ qui fera par la suite l'objet d'une révision en séance plénière, le Vice-Président, Me Claude Foisy, rappelle en premier lieu les tenants et aboutissants de la théorie du "Equal partnership" qui avait prévalu jusqu'à ce jour.

Essentiellement dans ces décisions le Conseil a décidé qu'à compter de l'accréditation, une nouvelle relation était établie et sur laquelle se fondait dorénavant le lien juridique employeur-employé. Le contrat individuel de travail était remplacé par un rapport ou relation collective. Le Conseil a, de plus, décidé que le droit de veto, consenti au syndicat dans l'article 148b), ajouté à la nouvelle relation qui était créée par l'accréditation, avait non seulement aboli le contrat individuel de travail, mais avait fait naître une association véritable (Equal partnership) entre le syndicat et l'employeur, et ce, pour la période de gel. Le Conseil a aussi continué en disant que ce gel imposé par l'article 148b) et l'association égale créé faisaient en sorte que, tant l'employeur que le syndicat devaient négocier à partir de zéro et consigner dans une convention collective les conditions qui régiraient dorénavant leur rapport collectif. Le Conseil a décidé que, tant que cette convention collective ne serait pas signée ou que le droit de grève ou de lockout ne serait pas acquis, l'employeur devait administrer son entreprise de concert avec le syndicat faisant en sorte que les droits de gérance résiduaires n'existaient plus, en d'autres mots, que le droit de gérance devait s'établir comme s'il n'avait jamais existé. En vertu de cette approche, l'article 148b) doit s'interpréter comme prohibant tout congédiement, mise à pied ou embauchage pour quelques raisons que se soient, et ce, pour la durée $d u$ gel, sauf, bien entendu, avec le consentement du syndicat. Cette interprétation de l'article 148b) est motivée par le désir d'amener le plus vite possible les deux parties négociantes, l'employeur et le syndicat, à une table de négociation et de les inciter à conclure rapidement une convention collective. Le raisonnement étant que l'employeur, privé de ses droits de gérance, et l'employé, de ses augmentations de salaire, tant l'un que l'autre voudront en finir au plus tôt.

14 Union internationale des employés de commerce et la Banque de Nouvelle-Écosse, Toronto, Ontario, (1981) 2 Can LRBR 365, 81 C.L.L.C. 16,110. 
Le deuxième motif sous-tendant cette interprétation de l'article 148b) cherche à éviter que l'employé reçoive des bénéfices décrétés unilatéralement par l'employeur, alors qu'on est en période de négociation collective. ${ }^{15}$

L'application de cette théorie avait pour effet de nier, à compter de l'envoi d'un avis de négocier à l'employeur, tous droits de gérance et de créer une association égale entre le syndicat et ce dernier. L'employeur ne pouvait par conséquent plus embaucher, congédier, faire de licenciement ou prendre des mesures disciplinaires, à moins que l'agent négociateur n'y consente. Or un syndicat nouvellement arrivé et dont l'un des premiers mandats est d'assurer la sécurité d'emploi peut difficilement se permettre, au vu et au su de ses membres, d'autoriser des congédiements et des mises à pied, même si ces derniers sont justifiés. D'autre part, même si l'agent négociateur voulait consentir à de telles modifications de conditions de travail il ne pourrait le faire valablement puisqu'il ne connaît pas les besoins de l'entreprise dont il vient d'hériter, selon cette théorie, de la moitié indivise. Par ailleurs, l'approche du gel total du droit de gérance obligerait l'employeur à faire d'importantes concessions en vue uniquement de pouvoir gérer son entreprise, de sorte que plutôt que de favoriser un climat propice à la négociation d'une première convention collective, l'employeur cherchera à acquérir le plus rapidement possible le droit au lock-out prévu à l'article 180a) à d) du Code en vue de reprendre son droit de gérance à part entière.

Cette interprétation de l'article $148 \mathrm{~b}$ ) rompait avec l'interprétation traditionnelle telle que suivie au Québec par le Tribunal du travail et en Ontario par le Ontario Labour Relations Board en vertu de laquelle, dans le contexte d'une première convention collective, le gel ne vise que les conditions de travail prévues au contrat individuel de travail. Il s'agit, en fait, de la théorie du «Statu quo" ou du "Business as usual" en vertu de laquelle l'employeur peut continuer à faire des mises à pied, des congédiements ou embauchages de nouveaux employés et à exercer son droit de gérance alors que l'employé peut continuer à recevoir les bénéfices de ses conditions de travail prévus à son contrat individuel de travail au moment de l'envoi de l'avis de négocier ou du dépôt d'une demande d'accréditation. En vertu de cette approche, si l'employeur avait coutume de donner une augmentation de salaire annuelle à l'employé, celui-ci doit la recevoir, même en période de gel, puisque cette coutume faisait partie de ses conditions de travail découlant de son contrat individuel de travail. Puisque cette approche a été retenue par le Conseil à l'occasion de plaintes formulées en vertu de l'article 124 (4) du Code et que le libellé de ce dernier est identique à celui de l'article 148b), le Conseil juge pour les mêmes raisons que l'obligation de l'employeur, à la suite d'un envoi d'un avis de négocier, est aussi semblable. Le Conseil justifie sa position de la façon suivante:

Si, suivant la jurisprudence, le contrat individuel de travail renaît ou se continue après l'expiration de la convention collective et que ses termes et conditions, dans ce cas jusqu'à ce qu'il soit changé, comprennent les termes et conditions contenus dans la convention collective expirée et qui sont de nature individuelle par opposition à syndicale et alors que la relation employeur-

15 Le texte français de la décision originale du Conseil dans cette affaire n'est pas encore rapporté. L'extrait rapporté ici est tiré des pages 8 et 9 de la décision du Conseil (dossier 745-740 (numéro 286)). La version anglaise peut être retrouvée, entre autre, à (1981) 2 Can LRBR 365 page 369. 
syndicat continue d'exister, ce dernier étant toujours accrédité et négociant le renouvellement de la convention collective et ce, même en période de grève ou de lock-out, il s'ensuit nécessairement que c'est la conclusion d'une convention collective, jusqu'à concurrence des termes y contenus, qui a abrogé au départ le contrat individuel de travail et non la relation dite collective et établie par l'accréditation. Si tel n'était pas le cas et que la relation basée sur l'accréditation fondait le lien juridique employeur-employé, le contrat individuel de travail ne renaîtrait pas à l'expiration de la convention collective car, jusqu'à la révocation de l'agent négociateur, la relation dite collective continue d'exister.

(...) Comme la relation juridique basée sur le contrat individuel continue d'exister jusqu'à la signature d'une première convention collective, et même jusqu'après son expiration, il nous apparaît difficile d'interpréter les termes de l'article 148b) qui ont trait aux changements des échelles de salaire, conditions, droits et privilèges des employés de façon différente à celle donnée à l'article $124(4) .16$

Il en résulte que ce qui est gelé, ce sont les conditions de travail contenues au contrat individuel qui existent au moment de l'avis de négocier, ou encore, la façon dont l'employeur et l'employé traitent au moment de l'avis.

Pour cette raison, le Conseil, constatant que la banque avait coutume d'accorder une augmentation salariale annuelle, juge que cette augmentation faisait partie des conditions de travail des employés des succursales pour qui l'avis de négocier avait été donné et que l'employeur avait modifié les conditions de travail des employés de ces dernières et ainsi violer les dispositions de l'article 148b) du Code canadien du travail en n'accordant pas ces augmentations aux employés syndiqués.

\section{RÉVISION EN SÉANCE PLÉNIÈRE}

Le Conseil, sous la plume de son vice-président Me Claude Foisy auquel se joint une majorité des membres, confirme l'approche du "Business as usual" adoptée dans la décision ci-dessus dans le cadre de l'application de l'article 148b) du Code. Puisqu'il avait déjà retenu cette doctrine dans le cadre de l'application de l'article 124 (4) du Code, il était logique qu'il conclût à l'application de la même doctrine suite à l'envoi d'un avis de négocier en vertu de l'article 146 du Code dans la mesure où l'accréditation par le Conseil, quoiqu'instaurant de nouveaux rapports juridiques dans l'entreprise, n'a pas pour effet d'abroger les contrats individuels de travail existants:

Mais d'une part, il est vrai d'affirmer que l'accréditation crée de nouveaux rapports juridiques dans l'entreprise, plus particulièrement entre l'employeur et le syndicat, par l'instauration d'un monopole de représentation syndicale.

16 Id., pages 20 et 21 ; pages 374 et 375 . Notons que l'une des différences fondamentales opposant les tenants de la théorie du "Equal partnership" et celle du "Business as usual" réside dans le fait que pour les premiers, l'accréditation accordée par le Conseil a pour effet d'abroger le contrat individuel de travail de même que de nier tout droit de gérance, alors que pour les seconds seule la conclusion d'une convention collective aura pour effet d'abroger les contrats individuels de travail et, ce, jusqu'à concurrence des termes y contenus. 
Cependant, d'autre part, nous ne pouvons conclure que l'accréditation ou encore l'envoi de l'avis de négociation anéantisse du coup tout ce qui a existé sur le plan contractuel entre un employeur et ses employés. En fait, ce n'est que lorsqu'une convention collective est signée que tout le cadre contractuel individuel "employeur-employé» se trouve à être vidé de son contenu. En effet, à notre avis il faut éviter de confondre les effets juridiques que procurent l'accréditation avec ceux qui découlent de la signature d'une convention collective. En d'autres termes, il existe une nette différence en droit entre le «pouvoir de représentation exclusif» et la "résultante de l'exercice de ce pouvoir de représentation exclusif».

(...) La «résultante principale de l'exercice de ce pouvoir de représentation exclusif» se retrouve dans les effets juridiques qui accompagnent la signature d'une convention collective entre l'employeur et l'agent négociateur. C'est à ce moment qu'un pas de plus est franchi dans le processus de changement amorcé par des employé qui ont décidé de donner un mandat à leur agent négociateur. La convention collective, sauf de rares exceptions, devient l'unique cadre de référence des relations employeur-employés concernant tous les sujets qu'elle traite. ${ }^{17}$

Le Conseil précise par la suite les effets de cette doctrine quant au droit de gérance de l'employeur suite à l'envoi d'un avis de négocier:

Avant d'aller plus loin, disons immédiatement que ce sont justement ces droits de gérance qui étaient consacrés par une pratique passée de l'employeur qui se trouveront gelés par l'effet du paragraphe $148 b)^{18}$.

En contrepartie, seront également gelées toutes les dispositions statutaires qui s'appliquent aux employés visés par l'envoi de l'avis de négocier, telles que les normes statutaires de travail, les dispositions de la Charte des droits, de même que tous les droits et privilèges dont jouissent les employés au moment de l'envoi dudit avis de négocier.

Le Conseil étudie enfin les avantages découlant de l'application d'une telle doctrine. Ainsi, du point de vue de l'employé, il n'est pas réaliste de penser qu'à compter du moment de l'émission du certificat d'accréditation, c'est-à-dire de la licence de négocier accordée au syndicat, ce geste du Conseil mettra toujours fin au climat d'incertitude, de méfiance et d'insécurité dans lequel se trouve l'employé ayant signé une carte d'adhésion syndicale. $\mathrm{Si}$, au surplus, en ayant recours à la doctrine du "Equal partnership" cet employé se voit privé d'une augmentation générale qu'en raison de la pratique passée les autres employés non syndiqués de l'entreprise reçoivent, il en résultera un effet dissuasif certain auprès des autres membres de l'entreprise ou du secteur dans lequel elle opère. Au contraire, l'application de la théorie du «Statu quo» permet à l'employé de recevoir une augmentation de salaire correspondant à la même que reçoivent les autres employés en fonction de la pratique établie, et ceci lui permet de pouvoir supporter sans trop en souffrir, l'attente, si elle devait être longue, avant la conclusion d'une convention collective.

Du point de vue du syndicat, la théorie du «Statu quo» déleste ce dernier de la pression qu'il subit habituellement lors de la négociation d'une première convention

17 Pages 7 et 8 de la décision en révision du Conseil.

18 Page 12 de la décision en révision du Conseil. 
collective en permettant que les employés puissent continuer de bénéficier des mêmes conditions de travail auxquelles ils auraient eu droit par la continuation de leur contrat individuel de travail et celles auxquelles ils sont habitués. Par ailleurs, si le syndicat est dans la position où l'employeur doit verser l'augmentation générale annuelle nonobstant le gel statutaire, il pourra alors négocier à partir de cette augmentation et, même s'il n'obtient rien de plus, il n'aura pas été mis dans la situation où il aura du la refuser. Un tel refus, au fur et à mesure que traîne en longueur la conclusion de la convention collective, devient de plus en plus difficile à justifier à l'égard - selon les termes du Conseil -, des néophytes que sont les nouveaux membres.

Enfin, du point de vue de l'employeur, la théorie du "Business as before" se justifie d'autant plus que, selon cette dernière, il ne sera pas privé de la totalité de ses droits de gérance. Au contraire, il pourra les exercer conformément à la pratique passée telle que gelée par l'effet de l'article 148b) du Code.

\section{Congédiement déguisé et émission explicite}

Le Conseil a jugé qu'il avait juridiction pour entendre une plainte de congédiement illégal présentée en vertu de l'article 184 (3)a) du Code par un employé ayant rédigé et remis à son supérieur une lettre de démission explicite.

Cette dernière, ayant été obtenue par suite de pressions indues de l'employeur, elle équivalait à un congédiement camouflé et la personne ayant fait l'objet d'un tel congédiement est un employé au sens du Code. Il y a par conséquent, dans de telles circonstances, ouverture à la juridiction du Conseil.

L'Union des employés de commerce, section locale 503, C.T.C. - F.T.Q., au nom de Carole Madeleine, plaignante, et Purolator Courier Ltée, Québec, employeur.

Dossier 745-1123; décision du 22 janvier 1982 (no 365); Panel du Conseil: Me Claude H. Foisy, Vice-Président, et Me Nicole Kean et Jacques Archambault, Membres.

La révision de cette décision en Cour fédérale, division d'Appel, a été demandée par la compagnie Purolator Courier Ltée en date du 29 janvier 1982 (dossier de la Cour no A-43-82).

Le Conseil énonce ainsi le critère eu égard auquel une démission, même explicite, devra être évaluée:

La question qui nous est posée par la présente affaire, dans le cadre de l'application de l'article 184 (3)a), est la même que celle considérée par les arbitres de griefs et nous sommes d'opinion qu'il y a lieu d'appliquer les principes que nous venons d'énoncer. En l'espèce, il s'agit d'un cas où Madame Madeleine a exprimé son intention explicite de démissionner en remettant à Monsieur Levert un écrit à cet effet. Il s'agit de se demander, si, eu égard aux circonstances particulières de la présente affaire, la démission, pour reprendre les temes de l'arbitre Dionne "a été donnée librement et volontairement et en toute connaissance des conséquences (car)... dès que les circonstances peuvent avoir vicié le consentement, il n'y a pas lieu de l'accepter ${ }^{19}$ ». $^{\prime}$

19 Page 34 de la décision du Conseil. 
Après avoir étudié les circonstances particulières de l'espèce et noté les pressions indues auxquelles avaient été soumises l'employée plaignante, le Conseil conclut de la façon suivante:

Nous concluons que Madame Madeleine n'a pas démissionné mais qu'elle a été condédiée. Dans ces circonstances, la plainte a été soumise par une employée au sens du Code et nous avons juridiction pour décider si l'article 184 (3)a) a été violé. Dans ces circonstances les faits allégués réfèrent à un congédiement et peuvent servir de base à une violation de l'article 184 (3)a ${ }^{20}$.

\section{ÉCOLE DE RELATIONS INDUSTRIELLES DE L'UNIVERSITÉ DE MONTRÉAL}

\section{Colloques}

- Formes nouvelles de syndicalisme, (5e colloque), 1974, 66 pages.

- L'État et la transformation des relations industrielles au Québec, (6e colloque), 1976, 64 pages.

- Les conflits en milieux de travail, (7e colloque), 1977, 100 pages.

- Pour une meilleure qualité de vie ... les avantages sociaux, (8e colloque), 1978, 96 pages.

- La réforme des lois du travail, (9e colloque), 1979, 124 pages.

- La transformation de l'entreprise et du travail. (10e colloque), 1980, 102 pages.

Monographies

- C. D'AOUST et L. LECLERC, La jurisprudence arbitrale québécoise en matière de congédiement, 1978,181 pages.

- L.P. DAUBIGNEY, Salaire et marché du travail interne d l'entreprise, 1978, 51 pages.

- G. GUERIN, Le système de planification des ressources humaines dans l'entreprise, 1978, 95 pages.

- S. DOLAN et A. ARSENAULT, Stress, santé et rendement au travail, 1980, 186 pages.

- C. D'AOUST et L. LECLERC, Les protocoles de retour au travail: une analyse juridique, 1980. 81 pages.

- G. HEBERT et J. VINCENT, L'environnement et le jeu des personnalités dans la négociation collective, 1980, 77 pages.

- C. D'AOUST et L. VERSCHELDEN, Le droit québécois de la responsabilité civile des syndicats en cas de grève illegale, 1980, 82 pages.

Service des publications, Ecole de relations industrielles.

Université de Montréal, Case Postale 6128, Montreal, H3C 3J7, (514)343-7312.

$20 \quad$ Page 38 de la décision du Conseil. 